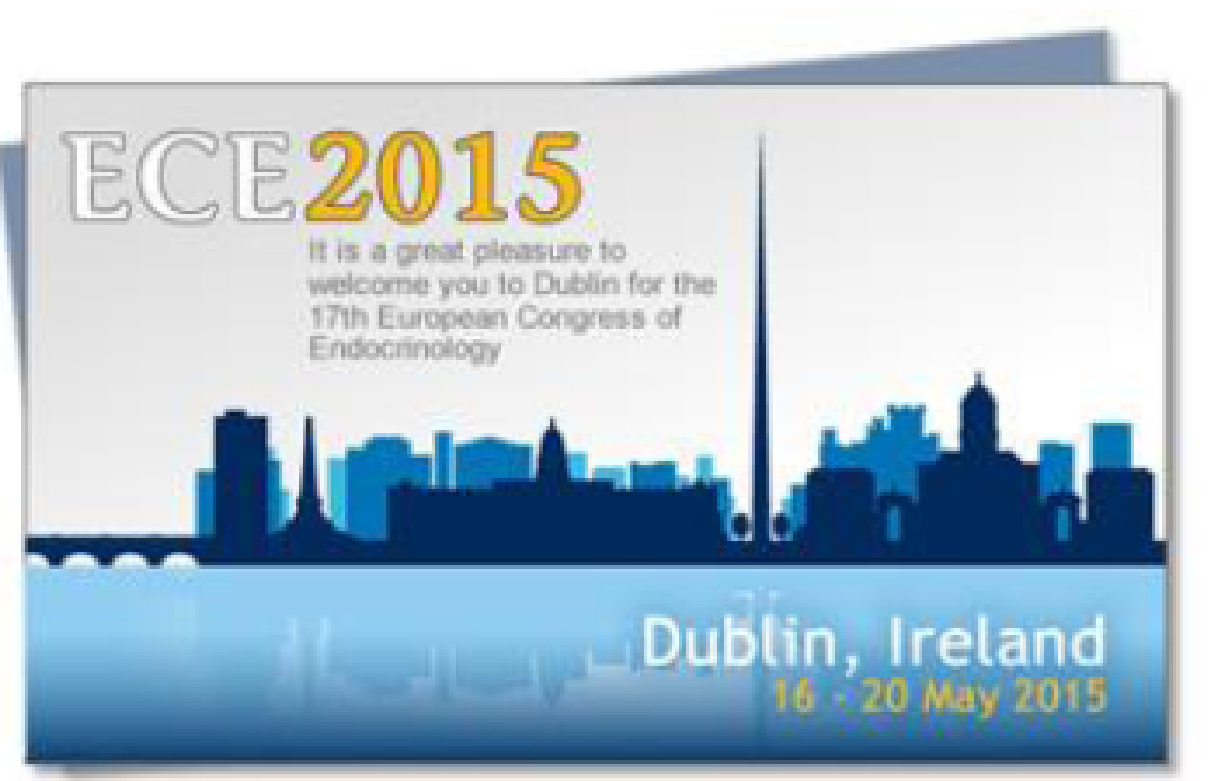

\title{
Salivary cortisol response to psychological stress in late adolescent and young women: impact of menstrual irregularity, hirsutism and hyperandrogenemia
}

\author{
Mezzullo M, Gambineri A, Fanelli F, Prontera O, Repaci A, Di Dalmazi G, Pagotto U and Pasquali R. \\ Endocrinology Unit, Department of Medical and Surgical Sciences (DIMEC), Center for Applied Biomedical Research (CRBA), S. Orsola-Malpighi University Hospital, \\ Alma Mater Studiorum - University of Bologna, Bologna, Italy.
}

Female hyperandrogenic disorders are known to arise in young age limiting the quality of life also due to psychological implication. Most epidemiological studies, however, have been carried out in the adult population, and little is known about their prevalence among adolescents and in young age. Self-collection of saliva for hormone testing allows to avoid painful venipuncture and bias coming from alteration of habitual daily activities. Salivary cortisol (SalF) testing was proved to be useful in the evaluation of acute stress responses. Liquid-chromatography tandem-mass spectrometry (LC-MS/MS) technology offers high sensitivity and specificity to properly assess low levels of salivary cortisol. The aim of this study was to investigate the variation in SalF response to a stressor event in adolescent and young adult females in absence or in presence of different features of clinical hyperandrogenism.

METHODS: We selected 170 drug-free females aged 16-19y from a cross-sectional epidemiological study (HYperandrogenic state in Adolescents Young Adults, HYAYA). Saliva was collected in the morning before and after a stressor event consisting in a physical examination by a trained physician for anthropometric data collection and hirsutism scoring, and in a structured interview about familiar and menstrual history. Blood was collected for complete biochemical and hormonal evaluation. SalF and serum total testosterone (TT) were assessed by LC-MS/MS. Delta SalF\% was calculated as [(SalFpost - SalFpre) / SalF_post] x 100. Subjects were subdivided according to the presence of menstrual irregularities (MI group, $\leq 10$ bleeding/year; $n=29$ ), isolated hirsutism (IH group, modified Ferriman-Gallwey score $\geq 8$; $\mathrm{n}=38$ ), isolated hyperandrogenemia (IHA group, TT>age/menstrual phase-specific cut-off assessed by LC-MS/MS'; $n=12$ ). Normal controls were defined by the absence of the previous described features (NC group, TT< age/menstrual phase-specific cut-off' ${ }^{1},>10$ bleeding/year, modified Ferriman-Gallwey score $<8$; $n=91$ )

RESULTS: General features of each groups are showed in Table $n$ 1, data are shown as means \pm standard error of mean (SEM). Glucose and lipid profiles were normal and not different among groups. Compared to NC $(21.2 \pm 0.3 \mathrm{~kg} / \mathrm{m} 2)$, IH $(22.4 \pm 0.5 \mathrm{~kg} / \mathrm{m} 2, \mathrm{p}=0.017)$ and IHA $(23.5 \pm 0.8 \mathrm{~kg} / \mathrm{m} 2, \mathrm{p}=0.004)$ displayed higher body mass index (BMI); IHA also displayed higher waist circumference $(75.4 \pm 0.71$ vs $81.0 \pm 2.3 \mathrm{~cm}$, respectively, $\mathrm{p}=0.015)$, higher hip circumference $(98.2 .4 \pm 0.1 .6$ vs $92.1 \pm 0.7 \mathrm{~cm}$, respectively, $p=0.003)$ and higher TT $(0.295 \pm 0.01$ vs $0.59 \pm 0.03 \mathrm{ng} / \mathrm{ml}$, respectively, $P<0.0001)$. Basal SalF was not different among groups ( $p=0.977)$ as showed in Figure $1 \mathrm{~A}$; compared to NC, a SalF increasing trend after the stressor event was observed only in IH $(1.21 \pm 0.15 \mathrm{vs} 1.67 \pm 0.23 \mathrm{ng} / \mathrm{ml}, \mathrm{p}=0.071)$ as showed in Figures $1 \mathrm{~B}$ and $1 \mathrm{D}$. Delta SalF\%, was significantly different among groups $(p=0.015)$; in particular, Delta SalF\% was significantly higher in IH compared to NC $(56.1 \pm 18.2$ vs $3.4 \pm 5.1 \%$, respectively, $p=0.010$ ), Figure $1 C$, and these data was confirmed after adjustment for BMI, SHBG and waist circumferences $(p=0.023)$.

\begin{tabular}{|c|c|c|c|c|c|}
\hline & 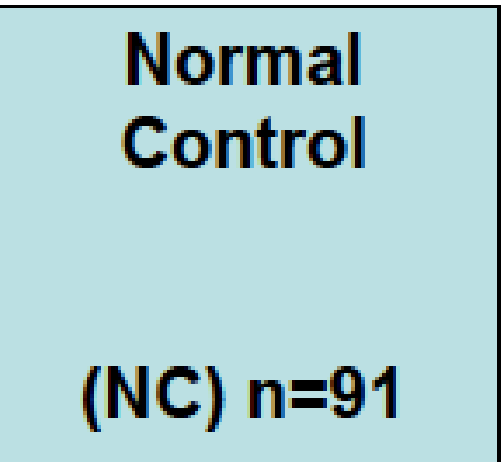 & $\begin{array}{c}\text { Isolate } \\
\text { Menstrual } \\
\text { Irregularities } \\
\text { (MI) } \mathrm{n}=29\end{array}$ & $\begin{array}{l}\begin{array}{c}\text { Isolated } \\
\text { Hirsutism }\end{array} \\
(\mathrm{IH}) \mathrm{n=38}\end{array}$ & $\begin{array}{l}\text { Isolated Hyper- } \\
\text { androgenemia } \\
\text { (IHA) } n=12\end{array}$ & ANOVA \\
\hline Age (years) & $17.4 \pm 0.1$ & $17.4 \pm 0.2$ & $17.7 \pm 0.1$ & $17.6 \pm 0.4$ & $P=0.224$ \\
\hline BMI $\left(\mathrm{Kg} / \mathrm{m}^{2}\right)$ & $21.2 \pm 0.3$ & $21.1 \pm 0.3$ & $22.4 \pm 0.5^{*}$ & $23.5 \pm 0.8$ * & $P=0.005$ \\
\hline Hip (cm) & $92.1 \pm 0.7$ & $92.2 \pm 1.3$ & $94.7 \pm 1.2$ & $98.2 \pm 1.6$ ** & $P=0.011$ \\
\hline Waist (cm) & $75.4 \pm 0.7$ & $73.8 \pm 1.2$ & $74.9 \pm 1.6$ & $81.0 \pm 2.3$ * & $P=0.050$ \\
\hline $\mathrm{DBP}(\mathrm{mmHg})$ & $70.4 \pm 1.0$ & $71.2 \pm 1.7$ & $70.3 \pm 1.4$ & $72.1 \pm 2.6$ & $P=0.938$ \\
\hline $\mathrm{SBP}(\mathrm{mmHg})$ & $113.3 \pm 1.2$ & $113.4 \pm 2.1$ & $113.7 \pm 2.1$ & $113.8 \pm 3.0$ & $P=0.979$ \\
\hline Insulin $(\mu \mathrm{U} / \mathrm{ml})$ & $7.65 \pm 0.30$ & $7.91 \pm 0.63$ & $8.81 \pm 0.77$ & $11.10 \pm 2.10$ & $P=0.026$ \\
\hline Glucose (mg/dl) & $75.1 \pm 1.2$ & $75.4 \pm 2.4$ & $80.6 \pm 1.7$ & $72.7 \pm 4.4$ & $P=0.081$ \\
\hline Triglycerides (mg/dl) & $67.3 \pm 2.2$ & $66.9 \pm 4.2$ & $69.0 \pm 5.1$ & $77.9 \pm 8.9$ & $P=0.511$ \\
\hline Colsterol tot $(\mathrm{mg} / \mathrm{dl})$ & $163.4 \pm 3.1$ & $170.8 \pm 5.7$ & $162.9 \pm 5.1$ & $178.6 \pm 10.6$ & $P=0.326$ \\
\hline $\mathrm{HDL}$ (mg/dl) & $60.8 \pm 1.1$ & $62.5 \pm 2.3$ & $59.4 \pm 2.3$ & $64.0 \pm 2.3$ & $P=0.656$ \\
\hline $\mathrm{LH}(\mathrm{mlU} / \mathrm{ml})$ & $8.01 \pm 0.88$ & $7.81 \pm 1.14$ & $11.20 \pm 3.11$ & $13.3 \pm 2.5$ & $P=0.274$ \\
\hline $\mathrm{FSH}(\mathrm{mlU} / \mathrm{ml})$ & $5.60 \pm 0.34$ & $4.65 \pm 0.46$ & $6.37 \pm 0.60$ & $5.25 \pm 0.53$ & $P=0.228$ \\
\hline Progesterone $(\mathrm{ng} / \mathrm{ml})$ & $1.94 \pm 0.41$ & $3.14 \pm 1.07$ & $3.08 \pm 0.71$ & $2.64 \pm 1.68$ & $P=0.422$ \\
\hline 17OH-Progesterone (ng/ml) & $0.764 \pm 0.555$ & $1.04 \pm 0.16$ & $0.983 \pm 0.113$ & $0.970 \pm 0.232$ & $P=0.150$ \\
\hline Testosterone (ng/ml) & $0.295 \pm 0.010$ & $0.295 \pm 0.017$ & $0.295 \pm 0.012$ & 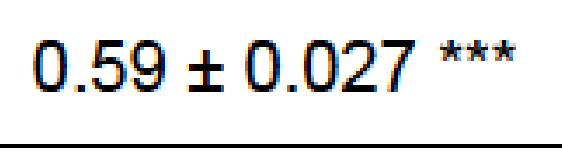 & $P<0.001$ \\
\hline DHEA (ng/ml) & $8.28 \pm 0.51$ & $6.75 \pm 0.54$ & $8.54 \pm 0.73$ & $8.29 \pm 1.04$ & $P=0.380$ \\
\hline Estradiol (pg/ml) & $106.1 \pm 11.4$ & $111.1 \pm 17.1$ & $99.7 \pm 16.6$ & $210.7 \pm 59.1$ & $P=0.022$ \\
\hline Cortisol (ng/ml) & $99.0 \pm 3.9$ & $97.5 \pm 10.2$ & $98.8 \pm 6.4$ & $93.1 \pm 5.9$ & $P=0.972$ \\
\hline SHBG (nmol/l) & $48.1 \pm 1.8$ & $53.5 \pm 5.8$ & $42.7 \pm 3.0$ & $52.9 \pm 12.3$ & $P=0.270$ \\
\hline Salivary F Pre (ng/ml) & $1.20 \pm 0.09$ & $1.39 \pm 0.30$ & $1.21 \pm 0.15$ & $1.09 \pm 0.21$ & $P=0.928$ \\
\hline Salivary F Post (ng/ml) & $1.19 \pm 0.11$ & $1.17 \pm 0.13$ & $1.68 \pm 0.23$ & $1.15 \pm 0.21$ & $P=0.097$ \\
\hline
\end{tabular}

Table 1: Anthropometric, metabolic and hormonal features of population

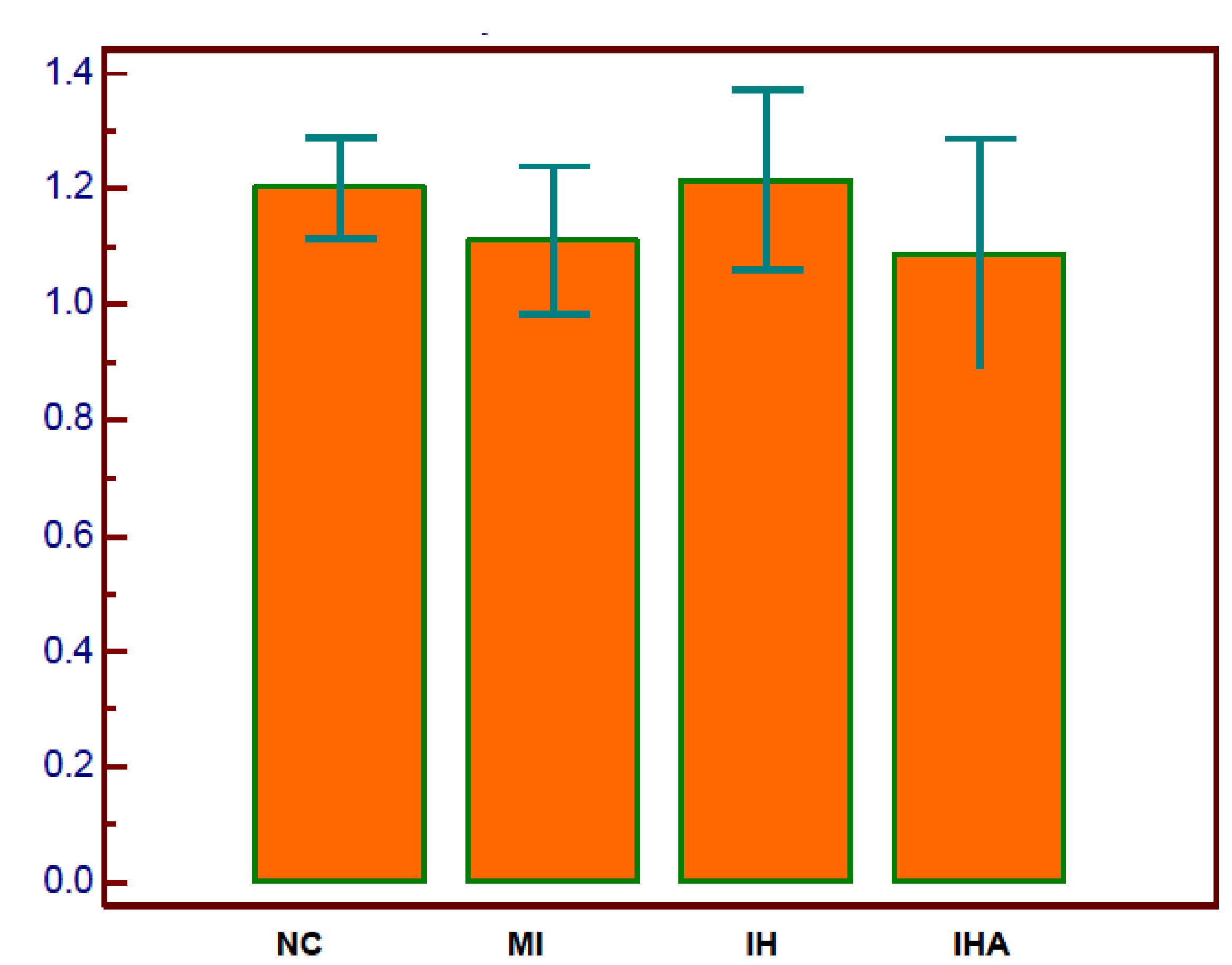

Figure 1A: Salivary cortisol levels $(\mathrm{ng} / \mathrm{ml})$ prior to phisical examination

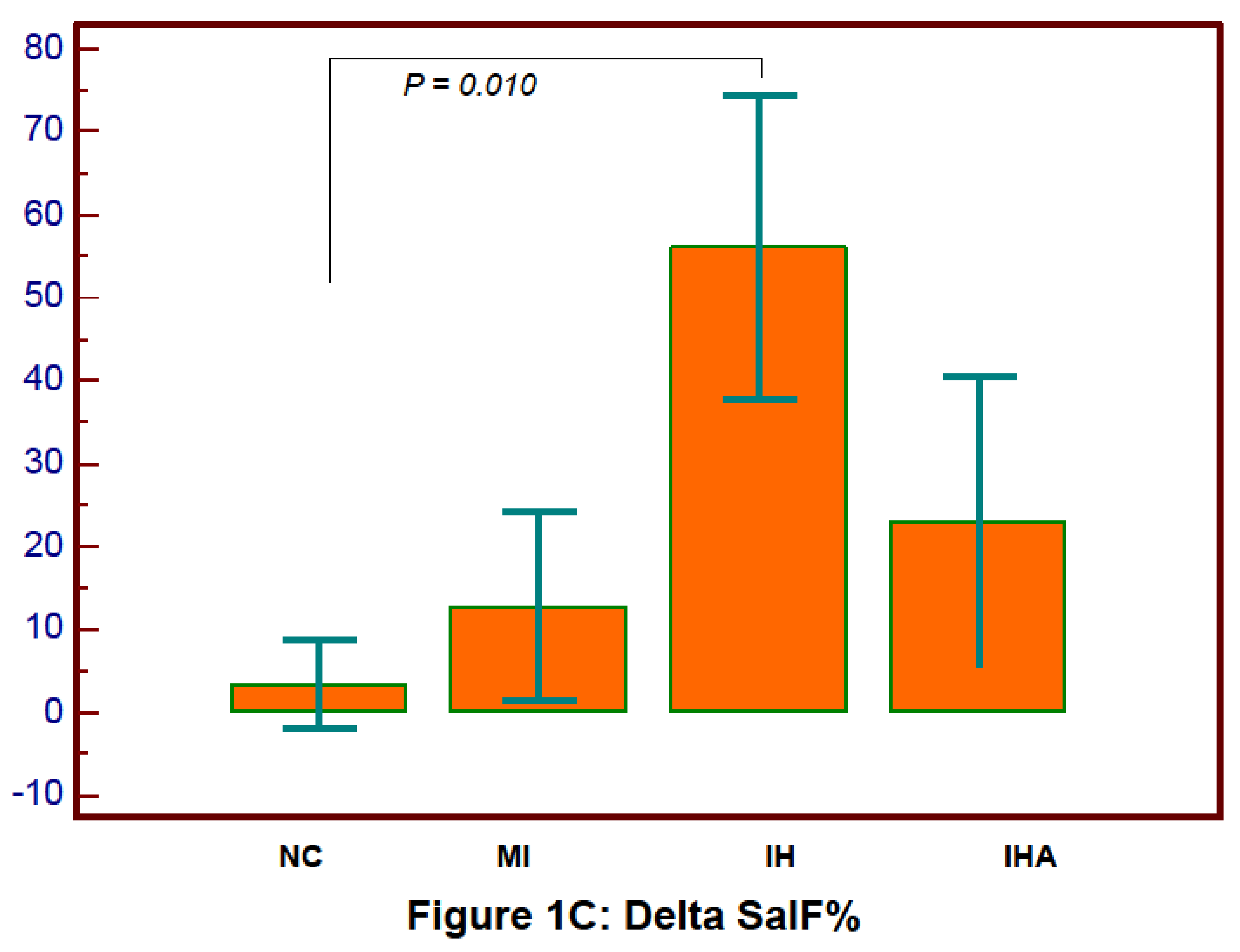

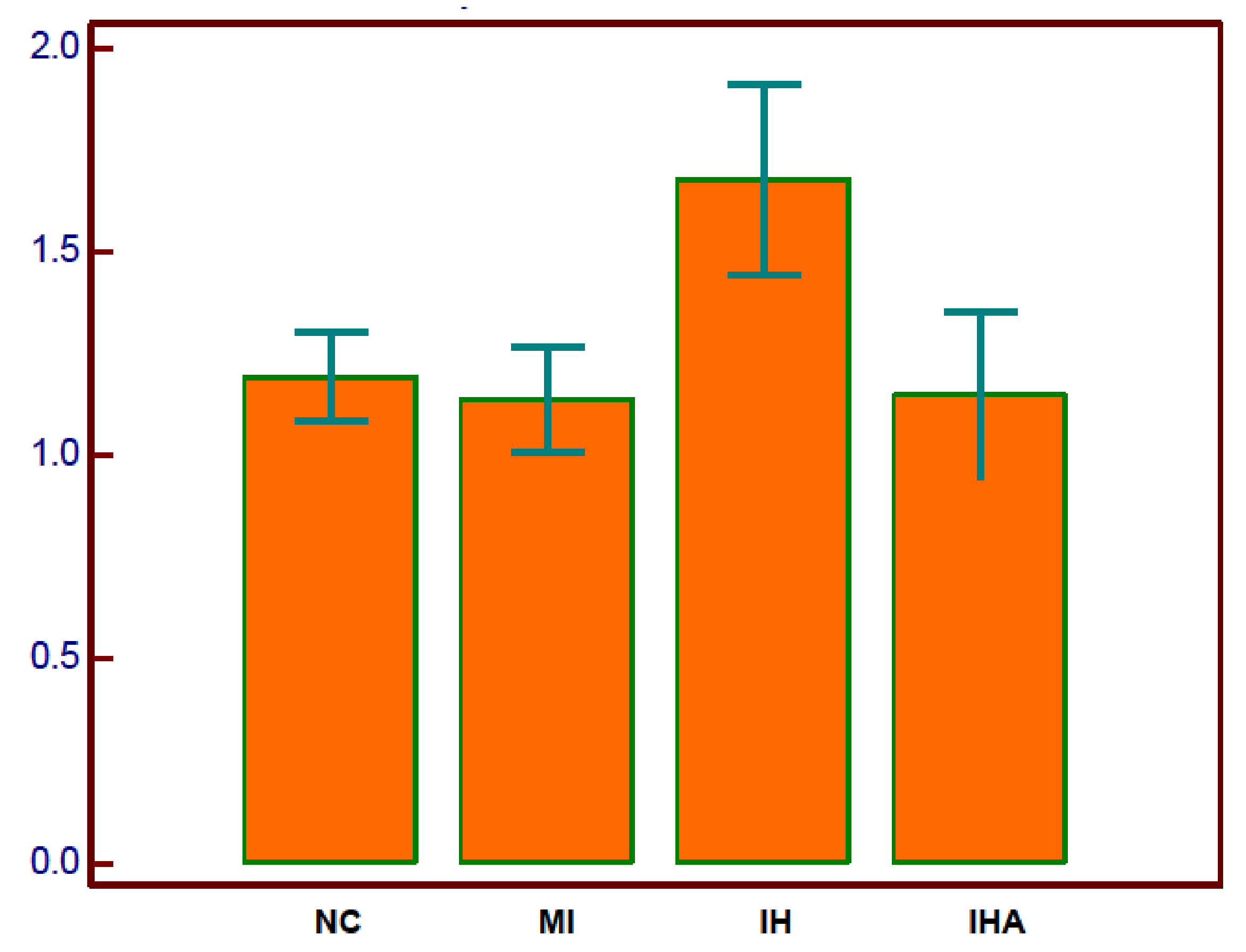

Figure 1B: Salivary cortisol levels (ng/ml) after physical examination

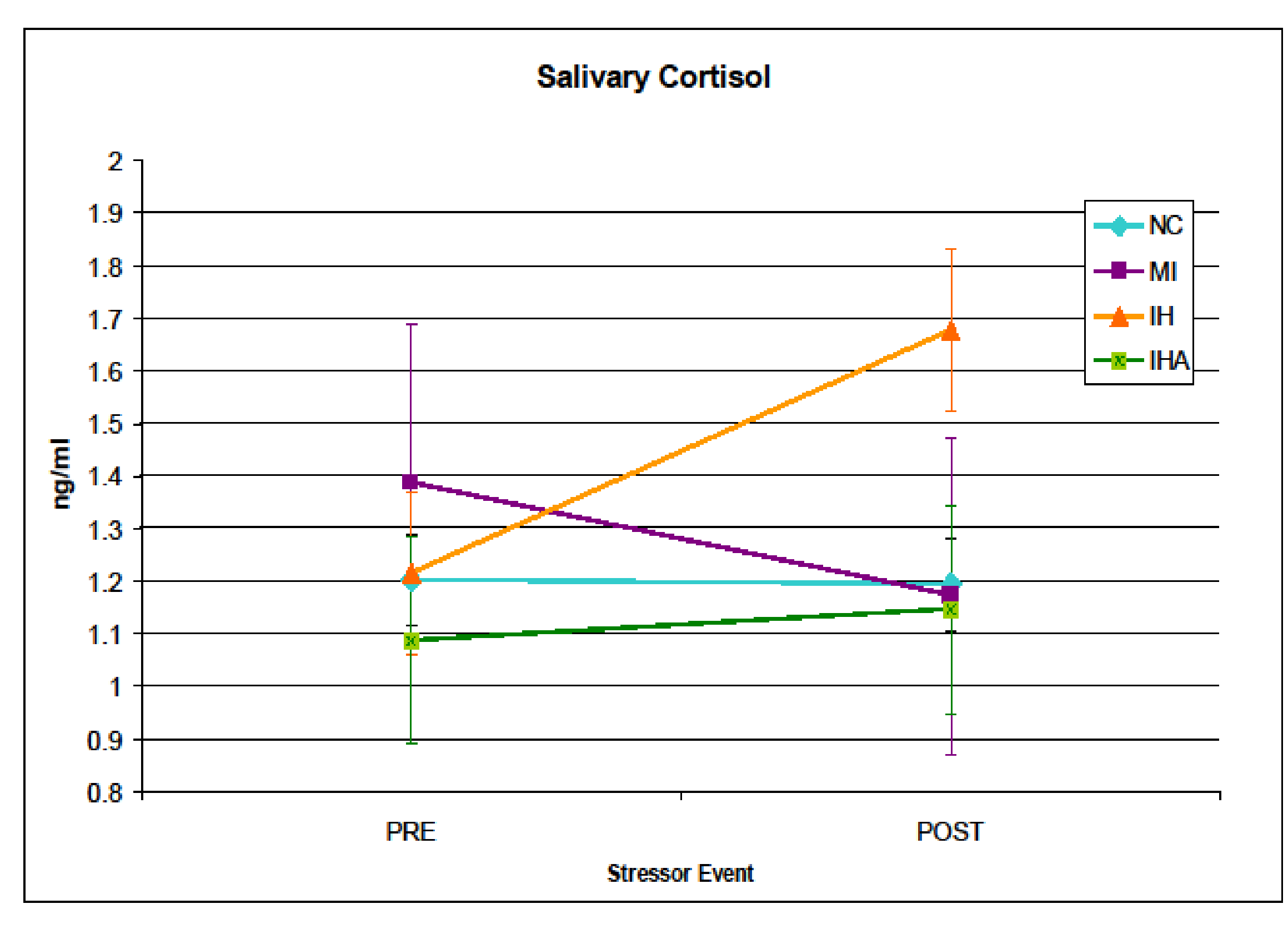

Figure 1D: Salivary F levels (ng/ml) pre stressor event vs post stressor event

CONCLUSION: We conclude that in our population of late adolescents and young women, hirsutism, a major feature of clinical hyperandrogenism, but neither isolated mestrual irregularities nor isolated hyperandrogenemia, plays a major role in the responsiveness to stress as measured by SalF. This finding highlights the importance of early recognizing psychological distress in such patients to promote psychosocial health and effective long term adherence to therapy. 